\title{
LETTERS
}

\section{Echocardiography of pulmonary vascular function in asymptomatic carriers of BMPR2 mutations}

\section{To the Editors:}

Pulmonary arterial hypertension $(\mathrm{PAH})$ is a rare, life-threatening dyspnoea-fatigue syndrome, caused by progressive increase in pulmonary vascular resistance (PVR) and eventual right ventricular failure [1]. The heritable form of PAH has been shown to be associated with mutations of the gene encoding the bone morphogenetic protein receptor-2 (BMPR2). Asymptomatic carriers of BMPR2 mutations are at high risk of developing $\mathrm{PAH}$ [2]. Careful follow-up of these subjects might help to detect early-stage disease with a more favourable response to targeted therapies. However, there is uncertainty about the optimal screening method. A recent European multicentre study showed that relatives of patients with idiopathic PAH (IPAH) present with an increased prevalence of abnormally high pulmonary artery pressure (PAP) during exercise or during low-oxygen breathing [3]. In that study, measurements of pulmonary vascular function were limited to a systolic PAP (sPAP) estimated from the maximum tricuspid regurgitation velocity (TRV) assessed by Doppler echocardiography. Here, we report on additional measurements performed in one of the participating centres, providing insight into abnormal pulmonary vascular distensibility and hypoxia-induced PVR in healthy BMPR2 carriers.

This study was part of a larger multicentre European project, which included 291 relatives of 109 IPAH patients and 191 agematched controls [3]. The participating PAH centre in Brussels, Belgium, contributed with 35 relatives of 10 index patients with IPAH and 38 healthy controls. The 35 relatives were aged mean \pm SD $35 \pm 14$ yrs. The 38 controls were aged mean \pm SD $36 \pm 10 \mathrm{yrs}$, and matched for sex and body surface area. Five of the asymptomatic relatives and two of the index IPAH patients were carriers of BMPR2 mutations. All relatives and controls underwent complete standard Doppler echocardiography at rest, followed by repetitive measurements of sPAP during exercise, with workload increased by $25 \mathrm{~W}$ every 2 min up to the maximum tolerated, and after $2 \mathrm{~h}$ of breathing an inspiratory oxygen fraction of 0.12 , as previously described [3]. sPAP was calculated from maximum TRV [4].

Additional analysis specific to this study consisted of right ventricular outflow tract time-velocity integral (RVOT VTI $_{\text {) }}$ during the resting normoxic and hypoxic measurements, to estimate a mean PAP (mPAP) from the acceleration time of pulmonary flow velocity [5], and PVR calculated as $10 \times \mathrm{TRV} /$ RVOT $_{\mathrm{VTI}}+0.16$ Wood units [6]. mPAP was also calculated from $\mathrm{sPAP}$ using the formula $\mathrm{mPAP}=0.6 \times \mathrm{sPAP}+2 \mathrm{mmHg}$ [7]. Cardiac output $(Q)$ was estimated from the left ventricular outflow tract (LVOT) diameter, LVOT $_{\text {VTI }}$ and cardiac frequency $(f C)$ at rest [8], and exercise-associated changes estimated from proportional changes in $f c$. Linear regressions were calculated on multipoint $\mathrm{mPAP}-Q$ plots, with adjustment for individual variability [9]. As multipoint $\mathrm{mPAP}-Q$ relationships actually disclose a slight curvilinearity, a distensibility coefficient $\alpha$ accounting for it was calculated using the equation $\mathrm{mPAP}=$ $\left(\left((1+\alpha \times \mathrm{LAP})^{5}+5 \alpha R_{0} Q\right)^{1 / 5}-1\right) / \alpha$, where $R_{0}$ is total PVR at rest and LAP is left atrial pressure, which is assumed to be unchanged from the resting state, as previously described [9]. Data are presented as mean \pm SD. The statistical analysis consisted of a repeated-measures ANOVA and modified paired t-tests when the F-ratio of the ANOVA reached a critical $p<0.05$ value.

The main results with measurements at rest, at the maximum workload achieved and after $2 \mathrm{~h}$ of hypoxia, are shown in table 1. Maximum workloads were $103 \pm 45 \mathrm{~W}$ in the BMPR2 carrier relatives, $113 \pm 38 \mathrm{~W}$ in the non-BMPR2 carrier relatives and $119 \pm 33 \mathrm{~W}$ in the controls. Resting mPAP calculated from TRV or from the acceleration time of RVOT $_{\text {VTI }}$ were not different: $14 \pm 2$ versus $12 \pm 6,15 \pm 2$ versus $14 \pm 3$ and $15 \pm 3$ versus $15 \pm 3 \mathrm{mmHg}$ in normoxia, and $28 \pm 5$ versus $29 \pm 5,27 \pm 5$ versus $25 \pm 6$ and $25 \pm 4$ versus $22 \pm 5 \mathrm{mmHg}$ in hypoxia in BMPR2 carrier relatives, non-BMPR2 carrier relatives and controls, respectively ( $\mathrm{p}$-values were always nonsignificant between the two methods). Blood pressure, $f C, \mathrm{mPAP}, Q, \mathrm{PVR}$ and the slopes of $\mathrm{mPAP}-Q$ relationships were not different between the three groups. PVR did not decrease during exercise in the BMPR2 carriers. Individual mPAP-Q relationships showed a marked interindividual variability (fig. 1). However, $\alpha$ was markedly decreased in the BMPR2 carriers, at rest and during exercise. Furthermore, the BMPR2 carriers also had an enhanced hypoxic pressure response when expressed as PVR versus arterial oxygen saturation measured by pulse oximetry. Hypoxia was associated with a decrease in $\alpha$, which was of the same magnitude in the three study groups. There was a correlation between $\mathrm{mPAP}$ after $120 \mathrm{~min}$ of hypoxia and at maximal exercise $(\mathrm{r}=0.49, \mathrm{p}<0.001)$.

The present results show the potential relevance of refined analysis of stress echocardiography PAP responses to exercise and hypoxia in terms of pressure-flow relationships and PVR$\mathrm{Sp}, \mathrm{O}_{2}$ plots. Previous invasive studies have shown that multipoint $\mathrm{mPAP}-\mathrm{Q}$ relationships conform to a distensible mathematical model of the pulmonary circulation better than the PVR equation [10]. Thus, adding a measure of the compliance of pulmonary resistive vessels allows for an improved prediction of pressures at given levels of flow. It is interesting that $\alpha$ calculated at $2 \%$ change in diameter per $\mathrm{mmHg}$ transmural pressure from invasively as well as noninvasively measured multipoint pressure-flow plots agrees with in vitro measurements in isolated arterioles $[9,10]$. Whether decreased $\alpha$ in the asymptomatic BMPR2 mutation carriers in the present study reflects early pathological changes with potential for progression is not known. 


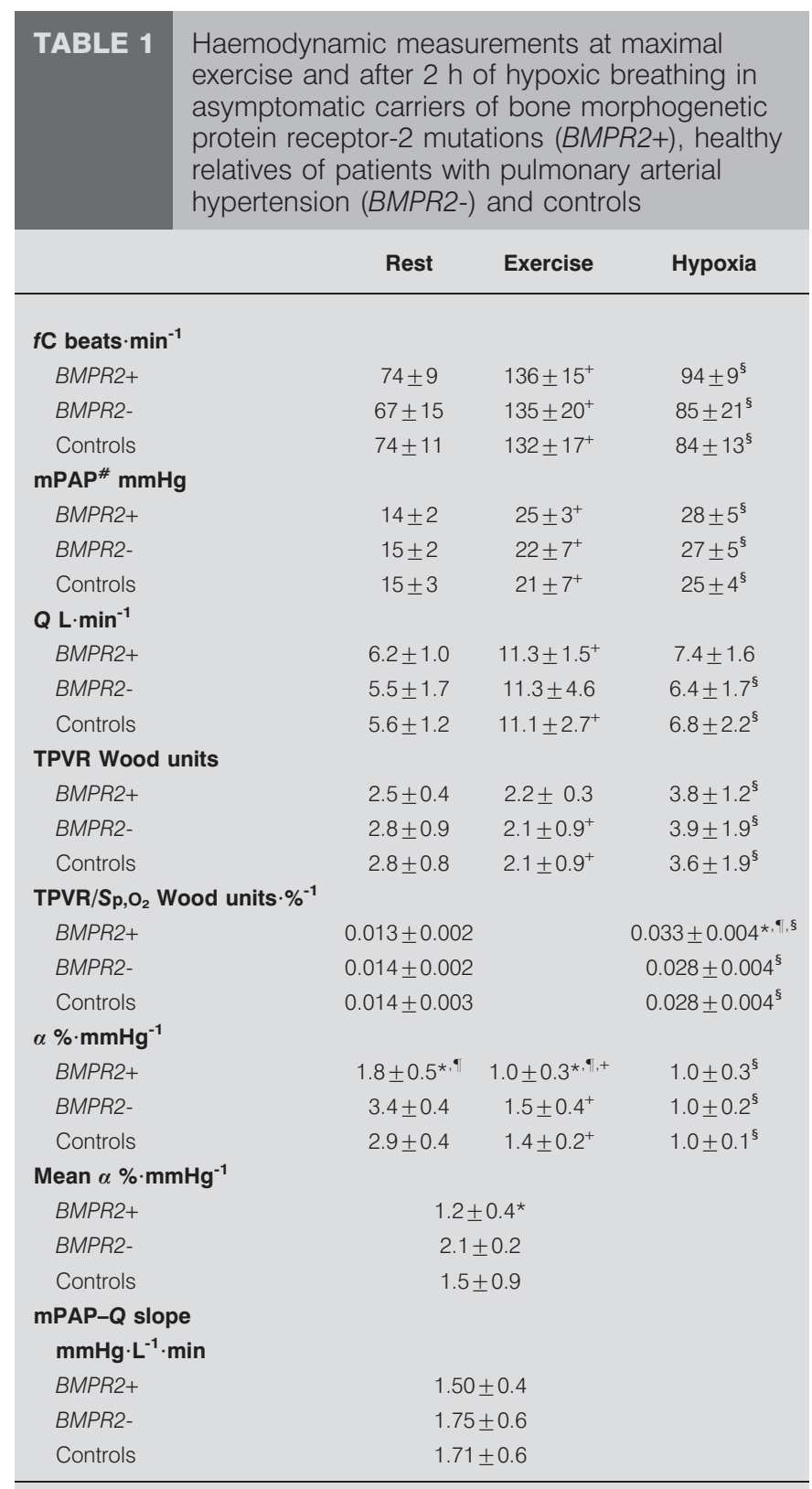

Data are presented as mean \pm SD. $f C$ : cardiac frequency; mPAP: mean pulmonary artery pressure; $Q$ : cardiac output; TPVR: total pulmonary vascular resistance; $\mathrm{Sp}_{\mathrm{O}} \mathrm{O}_{2}$ : arterial oxygen saturation measured by pulse oximetry; $\alpha$ : distensibility coefficient (in \% diameter change per $\mathrm{mmHg}$ pressure). \#: calculated from tricuspid jet velocity. *: $p<0.05$ for BMPR2+ versus BMPR2-;

$\because \mathrm{p}<0.05$ for BMPR2+ versus controls; ${ }^{+}: \mathrm{p}<0.05$ for exercise versus rest; s: $p<0.05$ for hypoxia versus rest.

Our data also show that healthy BMPR2 mutation carriers present with an enhanced hypoxic pressure response to hypoxia. This result agrees with recent report that BMPR2 mutant mice are more susceptible to hypoxic pulmonary hypertension [11]. Hypoxic breathing was associated with the expected decrease in $\alpha$ in the three study groups [10], suggesting that any intrinsic difference in pulmonary vascular compliance is overwhelmed by hypoxic pulmonary vasoconstriction.

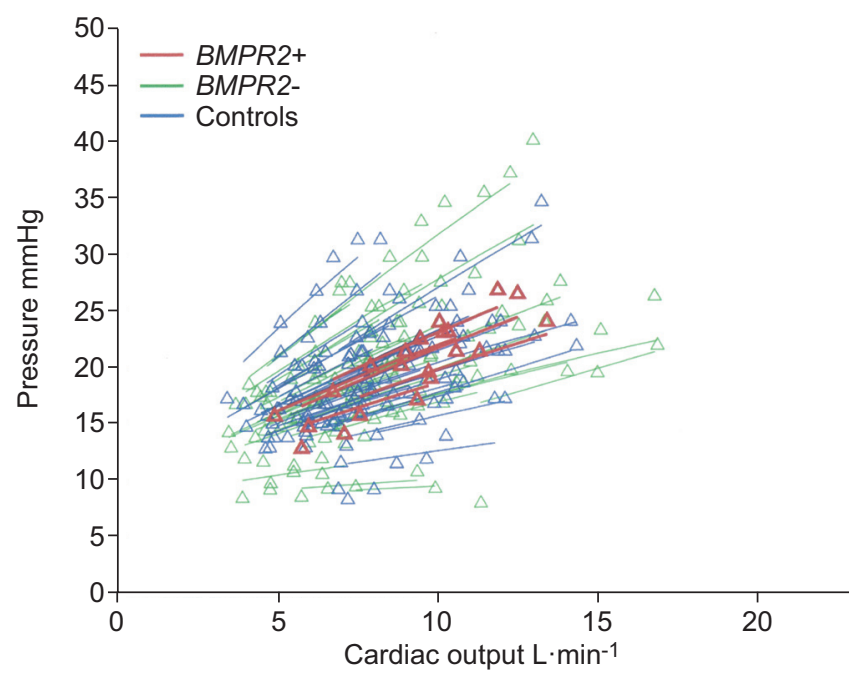

FIGURE 1. Individual multipoint mean pulmonary artery pressure-cardiac output plots, fitted using distensible models of the pulmonary circulation. The responses were variable, and similar in bone morphogenetic protein receptor-2 mutations (BMPR2+), healthy relatives of patients with pulmonary arterial hypertension (BMPR2-) and controls.

There are obvious limitations to the present preliminary report. The number of healthy BMPR2 mutation carriers was small, and pressure and flow calculations from noninvasive measurements rested on several assumptions. IPAH is a rare disease, with an incidence of one to two cases per million per year, only a minority of these patients is carrying a BMPR2 mutation, and asymptomatic relatives carrying a $B M P R 2$ mutation are difficult to identify [1-3]. Exercise stress echocardiographic estimates of PAP and $Q$ have previously been reported to be associated with realistic $\alpha$ calculations [9]. Left atrial pressure was not measured, but assumed constant, in keeping with previous estimates in normal subjects at exercise [9] and invasive studies showing that significant increases in LAP occur at exercise only at high workloads $>150 \mathrm{~W}$ associated with cardiac outputs over 15$20 \mathrm{~L} \cdot \mathrm{min}^{-1}$, much higher than in the present study [12]. The assumption in the present study that $f C$ would be the main determinant of exercise-induced increase in $Q$ may be more debatable. We therefore compared distensibility $\alpha$ and $Q$ calculated from changes in $f C$ only or from repetitive LVOT $_{V T I}$ and $f C$ measurements during exercise stress echocardiography in a total of 20 healthy subjects included in a recently reported study [13]. The results of both methods were highly correlated. The $f$ C-only method underestimated $Q$ only at values $>15 \mathrm{~L} \cdot \mathrm{min}^{-1}$ and $\alpha$ was underestimated by $\sim 10 \%$.

It is hoped that the present echocardiographic approach for the study of pulmonary vascular function will be applied with more direct measurements and further validation in future studies on patients with risk factors for the development of PAH.

\footnotetext{
Adriana Pavelescu*, Rebecca Vanderpool* , Jean-Luc Vachiéry ${ }^{\#}$, Ekkehard Grunig ${ }^{*}$ and Robert Naeije ${ }^{*, \#}$

*Dept of Pathophysiology, Faculty of Medicine, Université Libre de Bruxelles, and "Pulmonary Hypertension Clinic, Dept of Cardiology, Erasme University Hospital, Brussels, Belgium.
} 
"Center for Pulmonary Hypertension, Thoracic Clinic, University of Heidelberg, Heidelberg, Germany.

Correspondence: R. Naeije, Dept of Pathophysiology, Erasmus Campus, CP 604, Route de Lennik, 808, B-1070 Brussels, Belgium. E-mail: rnaeije@ulb.ac.be

Support Statement: This study was funded by a grant from the European Union Fifth Framework programme "Disposition to PPH, QLGI-CT-2002-01116".

Statement of Interest: Statements of interest for J-L. Vachiéry, E. Grunig and R. Naeije can be found at www.erj.ersjournals. $\mathrm{com} / \mathrm{site} / \mathrm{misc} /$ statements.xhtml

\section{REFERENCES}

1 Task Force for Diagnosis and Treatment of Pulmonary Hypertension of European Society of Cardiology (ESC), European Respiratory Society (ERS), International Society of Heart and Lung Transplantation (ISHLT), et al. Guidelines for the diagnosis and treatment of pulmonary hypertension. Eur Respir J 2009; 34 1219-1263.

2 Newman JH, Phillips JA, Lloyd JE. Narrative review: the enigma of pulmonary arterial hypertension: new insights from genetic studies. Ann Intern Med 2008; 148: 278-283.

3 Grunig E, Weissmann S, Ehlken N, et al. Stress Doppler echocardiography in relatives of patients with idiopathic and familial pulmonary arterial hypertension: results of a multicentric European analysis of pulmonary artery pressure response to exercise and hypoxia. Circulation 2009; 119: 1747-1757.
4 Yock PG, Popp RL. Noninvasive estimation of right ventricular systolic pressure by Doppler ultrasound in patients with tricuspid regurgitation. Circulation 1984; 70: 657-662.

5 Kitabatake A, Inoue M, Asao M, et al. Noninvasive evaluation of pulmonary hypertension by a pulsed Doppler technique. Circulation 1983; 68: 302-309.

6 Abbas AE, Fortuin FD, Schiller NB, et al. A simple method for noninvasive estimation of pulmonary vascular resistance. $J$ Am Coll Cardiol 2003; 41: 1021-1027.

7 Chemla D, Castelain V, Humbert M, et al. New formula for predicting mean pulmonary artery pressure using systolic pulmonary artery pressure. Chest 2004; 126: 1313-1317.

8 Christie J, Sheldahl LM, Tristani FE, et al. Determination of stroke volume and cardiac output during exercise: comparison of twodimensional and Doppler echocardiography, Fick oximetry, and thermodilution. Circulation 1987; 76: 539-547.

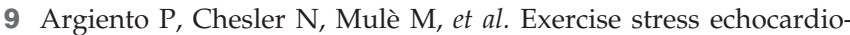
graphy for the study of the pulmonary circulation. Eur Respir J 2010; 35: 1273-1278.

10 Reeves JT, Linehan JH, Stenmark KR. Distensibility of the normal human lung circulation during exercise. Am J Physiol Lung Cell Mol Physiol 2005; 288: L419-L425.

11 Frank DB, Lowery J, Anderson L, et al. Increased susceptibility to hypoxic pulmonary hypertension in Bmpr2 mutant mice is associated with endothelial dysfunction in the pulmonary vasculature. Am J Physiol Lung Cell Mol Physiol 2008; 294: L98-L109.

12 Stickland MK, Welsh RC, Petersen SR, et al. Does fitness level modulate the cardiovascular hemodynamic response to exercise? J Appl Physiol 2006; 100: 1895-1901.

13 Groepenhoff $\mathrm{H}$, Overbeek MJ, Mulè M, et al. Exercise pathophysiology in patients with chronic mountain sickness. Chest 2012; [Epub ahead of print DOI: 10.1378/chest.11-2845].

DO|: $10.1183 / 09031936.00021712$

\section{Diabetes is a risk factor for tuberculosis in the Inuit population of Greenland}

\section{To the Editors:}

Diabetes is a known risk factor for tuberculosis (TB), and studies across populations and geographic regions suggest substantial increased risks of developing TB ranging from 1.16 to 7.83 with co-existing diabetes [1]. It is suggested that the risk is due to latent $\mathrm{TB}$ infections being activated by the hyperglycemia associated with diabetes. The evolving epidemiology of concurrent diabetes and TB in settings where both diseases are frequent is therefore looked upon with special concern. The prevalence of diabetes among the Greenland Inuit is high (10\%) and increasing [2]. Meanwhile, the TB incidence remains high with an estimated 180 cases per 100,000 suggesting ongoing TB transmission [3]. The aim of this study was to quantify the effect of diabetes on TB development among the Greenland Inuit.

We conducted a retrospective cohort study. Study participants comprised ethnic Inuit who participated in two previously conducted cross-sectional studies where diabetes status were assessed; Inuit Health in Transition Study (IHIT) [4] and the Greenland Population Study (B99) [2]. All study participants in
IHIT and B99 were randomly selected from the Civil Registration System (CRS) in order to represent the entire Greenlandic population. The CRS provides all citizens of Greenland with a unique identification number at birth, facilitating tracing of persons through all public registries. The CRS identifier enabled follow-up of study participants in the National TB register. The study was approved by the Ethics Review of Greenland.

Participants not previously diagnosed with diabetes (98\%) underwent a standard oral glucose tolerance test (OGTT). Participants were categorised with diabetes if previously diagnosed with diabetes or if fasting and 2-h blood glucose levels were $\geqslant 7 \mathrm{mmol} \cdot \mathrm{L}^{-1}$ and $\geqslant 11.1 \mathrm{mmol} \cdot \mathrm{L}^{-1}$, respectively. Weight and height were measured and body mass index (BMI) calculated. Age and sex of participants were determined from CRS data. Place of residence, divided into town or settlement, was likewise derived from the CRS registry.

TB is a mandatory notifiable disease in Greenland and all incident cases diagnosed by a medical doctor are recorded in the National TB register [5]. For the present study, all incident 